\title{
Original
}

\section{Modeling and Simulation of Swelling Behaviors of Clay Minerals Observed in Laboratory}

\author{
by \\ Sang-tae $\mathrm{KIM}^{\dagger}$, Jiro $\mathrm{Y}_{\text {Amatomi }}{ }^{\dagger}$ and Gento Mogi ${ }^{\dagger}$
}

\begin{abstract}
The modeling of swelling behavior is a necessary step to evaluate swelling minerals as sealing materials and to design an underground waste repository. The purpose of this study is to construct a mathematical model to describe the swelling behaviors of clay minerals and to develop a new FEM program in which a hydromechanically coupled procedure is incorporated to analyze stresses, displacements, uptakes, and volume expansions /pressure build-ups in swelling geological materials.
\end{abstract}

Key Words: Modeling, Swelling Behavior, Clay Mineral, Laboratory, Simulation

\section{Introduction}

Swelling phenomenon should be understood and analyzed when underground excavations are designed and opened in swelling rocks and/or swelling minerals are planned to be used as groundwater insulator of geological waste repository. It is usual that the swelling rocks or soils have adverse effects on stability and ground control, however, if they are used for sealing materials in a closed space, volume expansions caused by swelling must be favorable. The principal advantage of swelling is to fill cracks and any voids generated or potentially existing in surrounding rock mass and to make the insulating system of disposal sites more tight and secure by volume increases produced during water uptake process from unsaturated to saturated state. The hydromechanical characteristics of swelling clay must be clarified to utilize the advantageous aspects as sealing materials. In the present paper, we will propose a numerical procedure to analyze swelling behaviors and examine the developed hydromechanical coupled FEM program whose validity and applicability will be discussed by comparison of the calculated pressure build-up curves with those experimentally observed.

\section{Modeling of moisture characteristics}

\section{Water Potential}

The water potential of water in a porous medium represents the degree of restriction of water to solid matrixes and has a controlling influence on the mobility of water within the media. Also

Received January 29, 1999

† Dept. of Geosytem Eng., The University of Tokyo, 7-3-1, Hongo, Bunkyo-Ku, Tokyo, 113-8656, Japan. 
it can be regarded that pore water absorbed among solid matrixes is more restricted than free water. The water potential is defined as the total potential energy of pore water per unit mass and can be expressed by ${ }^{1)}$

$$
\phi=\phi_{\mathrm{m}}+\phi_{\mathrm{o}}+\phi_{\mathrm{g}}
$$

where $\phi_{\mathrm{m}},, \psi_{\mathrm{o}}$ and $\phi_{\mathrm{g}}$ are matrix, osmotic, and gravitational potentials, respectively. The matrix potential depends on the capillary pressure acting between water and solid surfaces, while the osmotic changes with the concentration of dissolved substance in the water and the gravitational is the function of position/elevation of the water. By comparison with state of free water in atmospheric condition, the total potential of restricted water in unsaturated pore spaces should be negative and can be rewritten as

$$
h=\phi+z
$$

where h, $\phi$ and $z$ are total, pressure, and gravitational heads, respectively. All these heads are described as potential energy of water per unit weight. The pressure head $\phi$ is negative in unsaturated state and usually replaced with suction (negative pressure) $\Psi$, which has the same magnitude of the pressure head but opposite sign. When in saturated state the pressure head $\phi$ is positive and equal to the water pressure divided by weight of unit volume of water, namely $\phi=p_{w} /\left(\rho_{w} g\right)$

\section{Moisture retention curve}

The moisture retention curve represents the relationship between the suction and the water content of porous media. The curve is the most important hydraulic characteristics of unsaturated solid, which can vary according to solid structures and physico-chemical properties of solid and water. The suction, which can be regarded as a function of water content, is calculated by using a mathematical formula such as the model of Van Genuchten ${ }^{2}$.

$$
\Psi\left(\theta_{w}\right)=\frac{1}{\alpha}\left[\left(\frac{\theta_{w}-\theta_{r}}{\theta_{s}-\theta_{r}}\right)^{-\frac{n}{n-1}}-1\right]^{\frac{1}{n}}>0
$$

where $\theta_{w}$ is the volumetric water content, $\theta_{s}$ and $\theta_{r}$ are the saturated and the residual volumetric water contents, respectively, while $\alpha$ and $n$ are parameters that should be determined experimentally. Figure 1 shows the moisture retention curve of the compacted bentonite that was used in the present study. The montmorillonite content and properties of the clay used are shown in Table 1.

\section{Hydraulic conductivity}

In order to analyze the seepage flow both in unsaturated and saturated solids, the hydraulic conductivity $k\left(\theta_{w}\right)$, which controls the flow of water through inter-pore channels, must bequantitatively known as the function of water content.

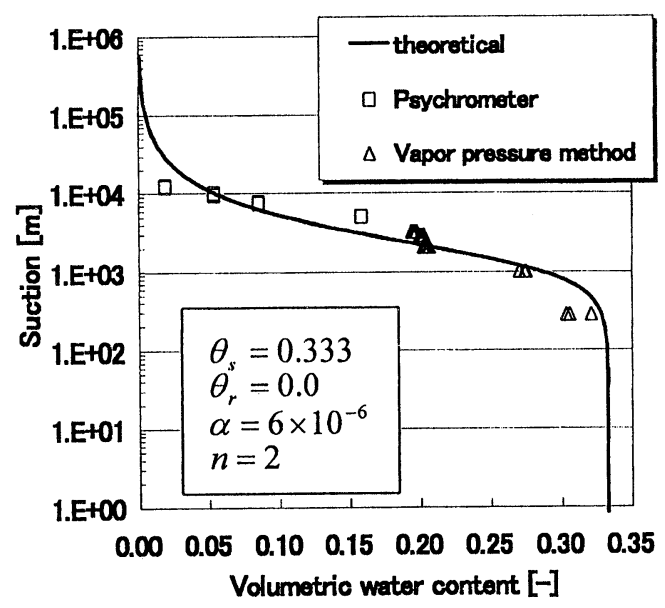

Figure 1 Moisture retention curve of bentonite ${ }^{3)}$ The Darcy's law can be applicable even in unsatu- 
rated state as follows

$$
q=-k\left(\theta_{w}\right)\left(\frac{\partial h}{\partial z}\right)
$$

It is, however, quite difficult to evaluate $k\left(\theta_{w}\right)$ because of the experimental difficulties in measuring local hydraulic gradients $\partial h / \partial z$.

To overcome this problem, the above equation should be rewritten as

$$
\begin{aligned}
q & =-k\left(\theta_{w}\right)\left(\frac{\partial \phi}{\partial z}+1\right) \\
& =-k\left(\theta_{w}\right) \frac{\partial \phi}{\partial \theta_{w}} \frac{\partial \theta_{w}}{\partial z}=-\frac{k\left(\theta_{w}\right)}{C\left(\theta_{w}\right)} \frac{\partial \theta_{w}}{\partial z}=-\mathrm{D}\left(\theta_{w}\right) \frac{\partial \theta_{w}}{\partial z}
\end{aligned}
$$

where $C\left(\theta_{w}\right)$ and $\mathrm{D}\left(\theta_{w}\right)$ are specific water capacity and water diffusivity, respectively, with the as sumption of $|\partial \phi / \partial z| \gg 1$. The water diffusivity can be expressed as the following experimental equation $^{3)}$.

$$
D\left(\theta_{w}\right)=D_{v}+D_{l}=\frac{a_{1}\left(\theta_{w}-\theta_{s}\right)}{\left(\theta_{w}-b_{1}\right)\left(b_{1}-\theta_{s}\right)}=\frac{a_{2} \theta_{w}}{b_{2}\left(\theta_{w}-b_{2}\right)}
$$

where $D_{v}$ is vapor diffusivity of water, $D_{l}$ is liquid diffusivity of water, in addition, $a_{1}, a_{2}, b_{1}$ and $b_{2}$ are experimentally determined parameters. Figure 2 represents the water diffusivity of the compacted bentonite and means that water flow in clay minerals can be considered as two-phase flow. Therefore, the hydraulic conductivity in unsaturated region can be calculated by following equation.

$$
k\left(\theta_{w}\right)=D\left(\theta_{w}\right) C\left(\theta_{w}\right)
$$

\section{Modeling of argillaceous swelling}

\section{Swelling Pressure}

Swelling is the volumetric expansion of a porous medium, which contains clay minerals and/or anhydrite, caused by water uptake. Of course, the mechanisms of swelling in both types of materials are completely different. The former is due to the widening of the inter space between clay layers generated by water uptake. The latter, on the other hand, is due to the hydration of anhydrite ${ }^{4}$. When anhydrite and water coexist within a closed system, anhydrite dissolves in water and precipitates in the form of gypsum, whose solid volume is about $61 \%$ larger than that of anhydrite. However, the chemical reaction and solidification of anhydrite are out of scope of the present investigation.

In argillaceous swelling, the ability to swell, which is expressed by swelling pressure $\pi$, can be related to suction $\Psi$ (i.e. negative pressure head in unsaturated state). In other way, the swelling 
pressure can be represented by the increase of water potential (or decrease of suction), as volumetric water content of swelling material increases as follows ${ }^{5)}$.

$$
\pi\left(\theta_{w}\right)=\int_{\theta_{w o}}^{\theta_{w}} \frac{\partial \Psi}{\partial \theta_{w}} d \theta_{w}=\left[\Psi\left(\theta_{w}\right)-\Psi\left(\theta_{w o}\right)\right]
$$

where $\theta_{\text {wo }}$ and $\theta_{\mathrm{w}}$ represent initial and present volumetric water contents, respectively.

\section{Swelling parameter}

Swelling pressure is never identical to swelling stress that can be measured by a loadcell in a laboratory swelling test as shown in Figure 3. Usually, the swelling stress actually measured is lower than the swelling pressure defined by equation (8). Microscopic reorientation and rearrangementof clay particles caused by interlayer swelling, existence of non-swelling grains working as obstacles, and so on must reduce the swelling pressure actually acting against the loadcell. Therefore, the measured swelling stress should be less than the internally acting swelling pressure. The swelling stress is considered to be the total stress and can be represented by the following equation ${ }^{5}$.

$$
\sigma_{i j}=\sigma_{i j}^{\prime}-F \pi\left(\theta_{w}\right) \delta_{i j}
$$

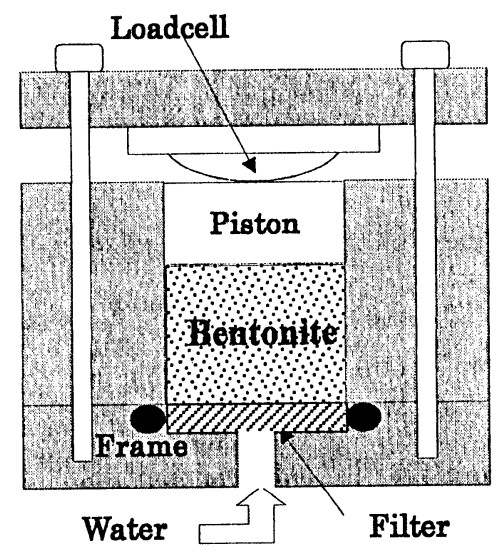

Figure 3 Schematic drawing of swelling test where $\sigma_{\mathrm{ij}}$ is the total stress, $\sigma_{\mathrm{ij}}^{\prime}$ is the effective stress, $\delta_{\mathrm{ij}}$ is the Kronecker's delta and $F$ is the swelling parameter, which should be evaluated by swelling experiments. There might be several possibilities to describe the swelling parameter as functions of water content or either the swelling parameter can be assumed constant. Since we cannot measure the true swelling pressure developed within clay interlayers, we assumed that the swelling parameter be an exponentially decreasing function of water content and a constant swelling parameter. Therefore, we have employed both assumptions and conducted simulations in order to identify which assumption is more favorite than the other.

\section{Governing equations for swelling analysis}

In order to clarify the water infiltration and associated swelling of clay minerals, we have had hydromechanically coupled analysis ${ }^{6)}$ ). Hydromechanically coupled processes must be governed by the constitutive equations of the stress-strain and the extended Darcy's law of fluid velocities as well as the equilibrium equation for stresses, the continuity equations, and the kinematic equations ${ }^{8) \sim 11}$.

Governing equations of swelling analysis can be ultimately integrated in the following equilibrium equations of the total stress, equation (10), and the continuity equation of the liquid, equation (11). The total stress of the equilibrium equations was considered the sum of the effective stress and the swelling pressure. The continuity equation of liquid was rewritten by using the extended Darcy's law in which the flux of liquid flowing through deformable porous media was considered as the fluid velocities relative to displacing solid matrixes. 


$$
\begin{aligned}
& {\left[\frac{1}{2} C_{i j k l}\left(u_{k, l}-u_{l, k}\right)-F \pi \delta_{i j}\right]_{, j}+\rho b_{i}=0} \\
& {\left[k\left(\theta_{w}\right)(\phi+z)_{, i}\right]_{, i}-\theta_{w} \frac{\partial u_{i, i}}{\partial t}=S^{*} \frac{\partial \phi}{\partial t}}
\end{aligned}
$$

where $u_{k}$ and $\phi$ are the displacements and pressure heads respectively, $C_{i j k l}$ are the moduli of elasticity, $b_{i}$ is the body force, $\rho$ is the bulk density of porous medium and can be defined as

$$
\rho=(1-n) \rho_{s}+\theta_{w} \rho_{w}
$$

where $n$ is the porosity, $\rho_{s}$ and $\rho_{w}$ are the densities of solid and liquid respectively. $S^{*}$ is the specific volume storativity can be defined by either equations (13a) or (13b); the former is applicable in unsaturated zone and the latter for capillary fringes and saturated zone,

$$
S^{*}= \begin{cases}\theta_{w} \beta_{w} \rho_{w} g+C(\phi) & \text { (a) } \\ \rho_{w} g\left[n \beta_{w}+(1-n) \beta_{b}\right] & \text { (b) }\end{cases}
$$

where $g$ is the acceleration of gravity, $\beta_{b}$ and $\beta_{w}$ are the compressibilities of solid matrixes and liquid, respectively.

\section{Numerical Results}

Figure 4 shows the mesh model and the boundary conditions. The mesh model has 539 nodes and 480 elements and is considered to be an axisymmetric body of revolution. As for the boundary conditions on the displacements and heads, the displacements on three sides are restricted and only the bottom boundary is allowed to contact pure water. Table 3 shows the properties of the specimen used for calculation, and they have been determined by laboratory experiments.

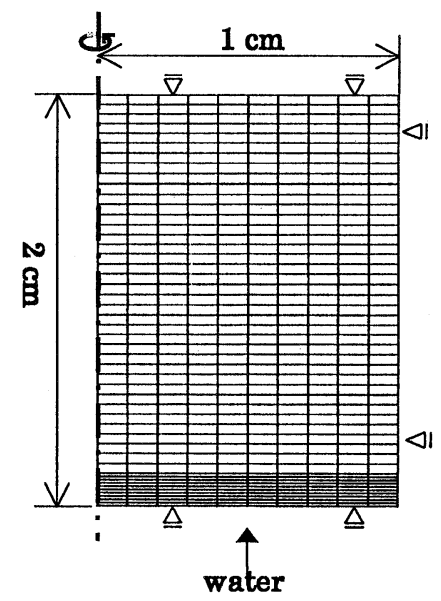

Table 3 Properties of specimen

\begin{tabular}{|c|c|}
\hline Type & Bentonite \\
\hline Dry density & $1.8 \times 10^{3}\left[\mathrm{~kg} / \mathrm{m}^{3}\right]$ \\
\hline $\begin{array}{c}\text { Saturation hydraulic } \\
\text { conductivity }\end{array}$ & $4.0 \times 10^{-14}[\mathrm{~m} / \mathrm{sec}]$ \\
\hline $\begin{array}{c}\text { Initial volumetric water } \\
\text { content }\end{array}$ & $0.16[-]$ \\
\hline $\begin{array}{c}\text { Final volumetric water } \\
\text { content }\end{array}$ & $0.333[-]$ \\
\hline Elastic modulus & $\begin{array}{c}1140-3091 \times \theta_{w}[\mathrm{MPa}] \\
\text { at } \theta_{r} \leq \theta_{w} \leq \theta_{s}\end{array}$ \\
\hline Poisson ratio & $0.3[-]$ \\
\hline
\end{tabular}

Figure 4 Mesh model

Figure 5 shows the movement of the moisture profiles caused by the upward infiltration of water with time. The volumetric water content at each element increased gradually from the initial value of 0.16 to the final value of 0.333 . Figure 6 shows the change of the effective stress with time caused 
by swelling mobilized by water uptakes. In Figure 6 , the effective stresses of the lower part in the specimen were positive/tensile at first; within the lower part, the local volume expansion was generated by the interlayer swelling pressure, while those of the upper part remained negative/compressive at initial stage (until 2 hours from starting water infiltration). After about 150 hours, the effective stresses of the lower part, which were compressed by the delayed expansion of the upper part, became negative/compressive. Finally, the effective stresses of the whole specimen turned compressive, since the swelling pressure was forced built up under the restricted boundary conditions after about 350 hours. Figure 7 is the comparison of the total stress (i. e. swelling stress) variation between the calculated and the experimental results measured by the loadcell on the top of the specimen. Even though the swelling stress built-up was successfully reproduced by the simulations, the numerical result assuming the constant swelling parameter is quite lower than the experimental data within the first 300 hours. On the contrary, a family better result has been obtained by using the swelling parameter represented as an exponential function of water content. It can be concluded that the apparent loss of swelling pressure generated by the microscopic reorientation and rearrangement of clay particles must be considered to decrease with the increase in water content during the process of swelling from the unsaturated to the saturated state in clay minerals.

\section{Conclusion}

In this study, the hydraulic and mechanical characteristics of the compacted bentonite during swelling were discussed by the numerical results obtained by the newly developed FEM program in which the water infiltration and the swelling phenomenon in clay materials can be simultaneously analyzed by employing a hydromechanically coupled procedure. In order that swelling pressure must

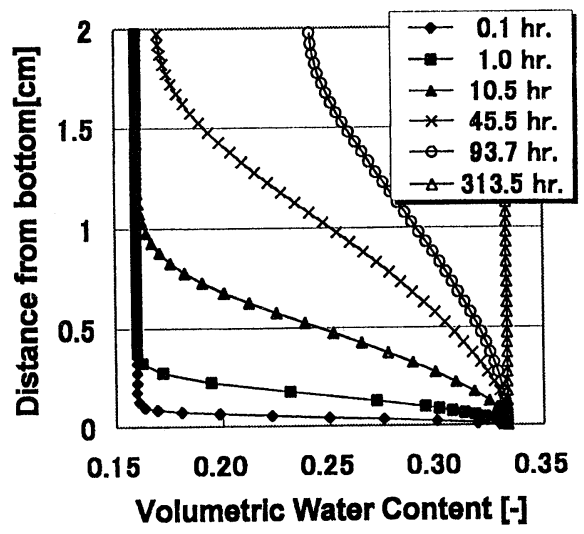

Figure 5 Changes of the moisture profiles caused by upward infiltration of water with time

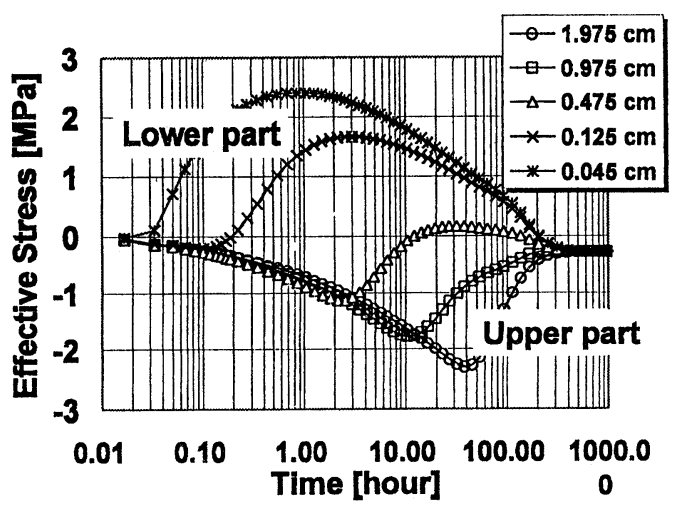

Figure 6 Changes of effective stress caused by swelling with time

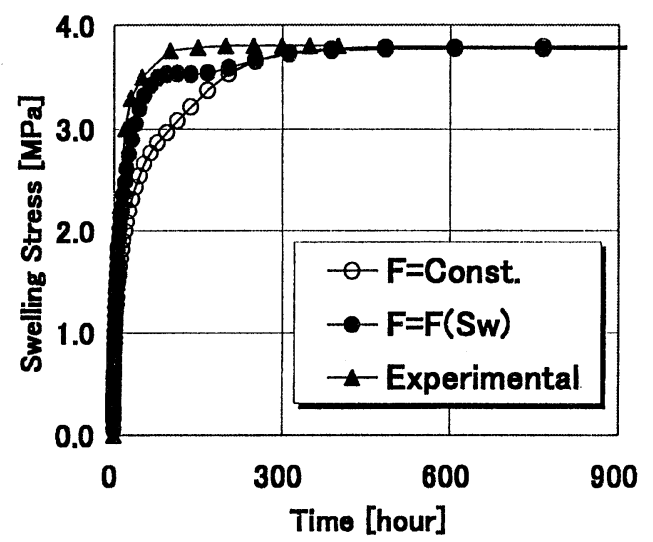

Figure 7 Comparisons of the experimental results of swelling test and the calculated results of two cases of swelling parameter 
be distinguishe from swelling stress, the swelling parameter was introduced. Since, in practice, it is quite difficult to define the swelling pressure mathematically, we haveassumed that the swelling parameter decreases exponentially with increase in moisture content and obtained more consistent swelling pressure build-up curve than using a constant swelling parameter.

\section{Acknowledgements}

The present study was supported by the Grant-in-Aid for Scientific Research from Ministry of Education, Science, Sports and Culture of Japan (International Scientific Research Program \#10044123).

\section{References}

1) Masahi. Nakano, "The movement of materials in soils", University of Tokyo, pp. 169-172 (1991). (In Japanese)

2) Van Genuchten, M. Th., "A closed-form equation for predicting the hydraulic conductivity of unsaturated soils", Soil Sci. Am. J. Vol. 44, No. 5, pp. 892-898 (1980)

3) JNC, "Water potential and water diffusivity of buffer material”, PNC TN8410 96-117 (1996). (In Japanese)

4) G.Anagnostou, "A model for Swelling Rock in Tunnelling”, Rock Mech. Rock Eng., 26 (4), $307-$ 331 (1993)

5) T., Fujita, M., Chijimatsu, T., Kanno, "Consideration on modeling of swelling stress of buffer material", Proceedings of the 50th annual conference of JSCE, pp. 28-29 (1995). (In Japanese)

6) Koichi Akai, Yuzo Ohnishi, Takeshhi Murakami, Masakuni Horita, "Coupled stress flow analysis in saturated-unsaturated medium by finite element method", $3^{\text {rd }}$ Int. Conf. on Numerical Methods in Geomech., pp. 241-249 (1979)

7) Yuzo Ohinishi, Hiroaki Shibata, Akira Kobayashi, "Numerical technique for analysis of coupled thermal-hydraulic-mechanical problem by finite element method", Proceedings of JSCE, Vol.370, III-5, pp. 151-158 (1986). (In Japanese)

8) Takuo Yamagami, "Some considerations on the governing equations of flow through deformable porous media", Proceedings of JSCE, Vol. 304, pp. 95-104 (1980). (In Japanese)

9) Ghislain de Marsily, "Quantitative hydrogeology", Academic press, Inc., pp. 85-114 (1986)

10) Peter S. Huyakorn, George F. Pinder, "Computational methods in subsurface flow", Academic press, Inc., pp. 229-257 (1983)

11) George F. Pinder, William G. Gray, "Finite element simulation in surface and subsurface hydrology", Academic press, Inc., pp. 127-201 (1977) 